\title{
Yogatherapie in der Schwangerschaft
}

Autorin: Hedwig H. Gupta

\section{ZUSAMMENFASSUNG}

Studien belegen, dass Yoga Frauen während der Schwangerschaft physisch und psychisch unterstützt. Es kann zur Prophylaxe eingesetzt werden, z. B. von Rückenschmerzen. Als Therapie bietet Yoga eine sichere und effiziente Option in allen Phasen der Schwangerschaft, beispielsweise bei Adaptationsstörungen, Gestosen, drohendem Abort, pränataler Depression bis hin zum Gestationsdiabetes. Wichtig ist die Auswahl und Anpassung der yogischen Techniken an die individuelle Situation der schwangeren Frau.

Gemäß dem Konzept der ayurvedischen Medizin wird die Yogatherapie von Empfehlungen zu Ernährung und Lebensstil begleitet.

\section{Schlüsselwörter}

Yoga, Yogatherapie, Ayurveda, Schwangerschaft, Geburt, Atmung, Asanas, Achtsamkeit

\section{ABSTRACT}

Studies show that yoga supports women during pregnancy physically as well as mentally. It can for example be used for the prophylaxis of backache. As a therapy, yoga offers a safe and efficient option during all phases of pregnancy, for example in the case of adjustment disorders, gestoses, imminent abortion, prenatal depression as well as gestational diabetes. The selection and adaptation of yogic techniques to the individual situation of the pregnant woman is important. According to the concept of Ayurvedic medicine, yoga therapy is accompanied by recommendations on nutrition and lifestyle.

\section{Keywords}

Yoga, yoga therapy, Ayurveda, pregnancy, birth, breathing, asanas, attentiveness

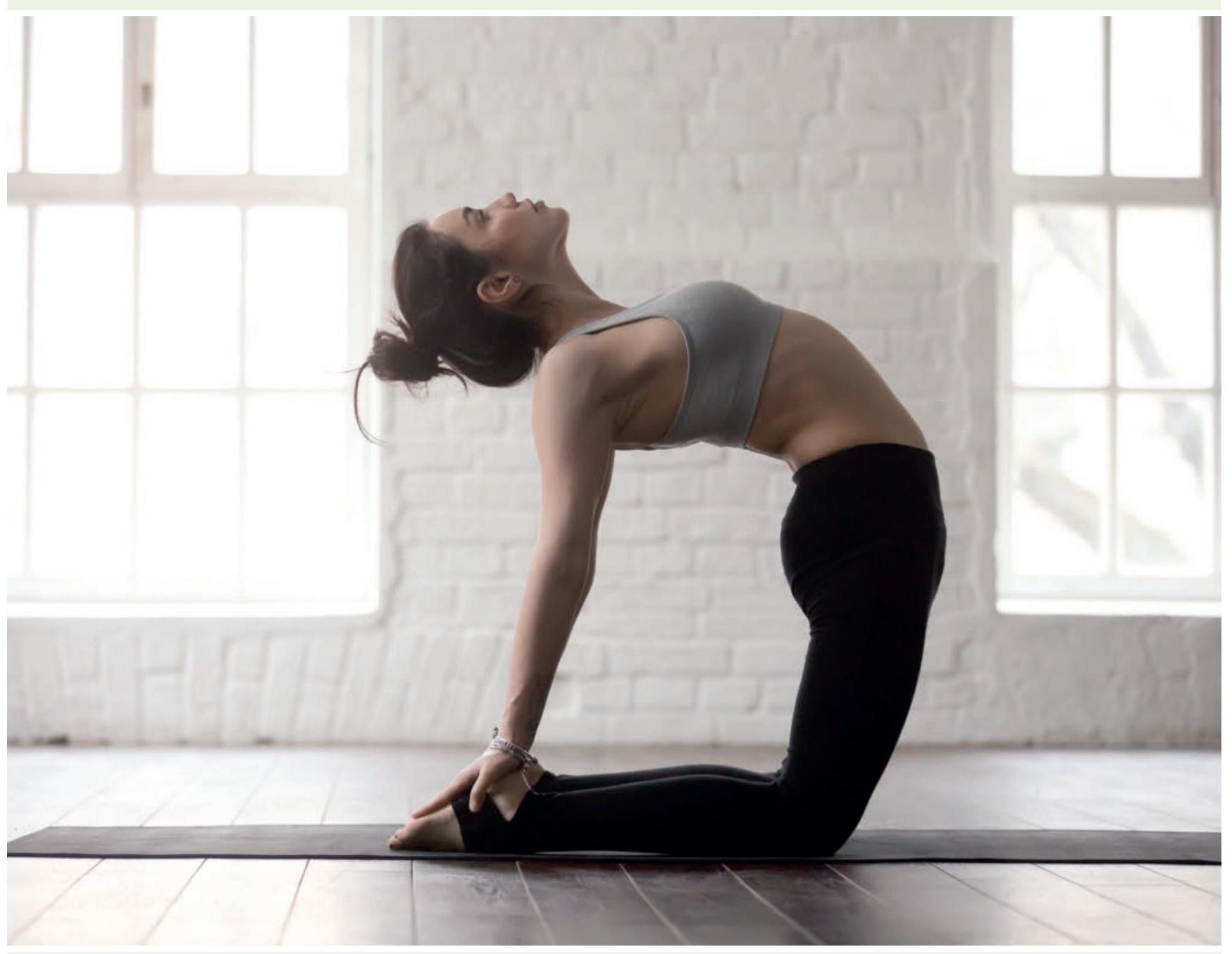

Abb. 1 Eine mögliche Übung für das erste Trimenon: Ushtrasana, das Kamel, kräftigt die Rumpf- und Beckenbodenmuskulatur und kann Rückenschmerzen vermeiden helfen. ๑ fizkes/Adobe Stock; nachgestellte Situation 
Die Schwangerschaft bedeutet für die werdende Mutter eine große Umstellungsleistung. Sie passt sich in einem extremen Maß dieser Sondersituation an. Das Herzkreislaufsystem wird durch die Gewichtszunahme, die Steigerung des Blutvolumens, den sinkenden peripheren Widerstand, die Eisenmangelanämie und die Abnahme des kolloidosmotischen Drucks belastet. Das Atemsystem wird durch den vermehrten $\mathrm{O}_{2}$-Bedarf und den Zwerchfellhochstand in späteren Schwangerschaftsstadien herausgefordert. Der Stoffwechsel wird durch Veränderungen der Sekretion von Thyroxin, Parathormon, Kortisol und Aldosteron bei anfangs erhöhter, später sinkender Insulinempfindlichkeit destabilisiert. Auch alle anderen funktionellen Systeme adaptieren sich stark an die Schwangerschaft und können in eine Fehlfunktion geraten.

\section{Ayurvedisches Verständnis der Schwangerschaft}

Auch aus ayurvedischer Sicht ist die Schwangerschaft eine sensible Periode im Leben der werdenden Mutter und ihrem Kind. Das dosha-Gleichgewicht wird belastet, insbesondere apana-vayu wird in der Frühschwangerschaft blockiert und in udana-vayu umgeleitet. Dies erklärt die Übelkeit und das Erbrechen in der Frühschwangerschaft gekoppelt mit einer Obstipationsneigung. Die agnis, die Verdauungsfeuer, müssen sich anpassen, um einen verstärkten Stoffwechsel leisten zu können. Es kommt leichter zu ama-Bildung, der Bildung von unvollständig abgebauten Metaboliten, was zu Störungen des Flusses in den funktionellen Systemen (srotamsi) führt. Dies kann sich in Ödemen, Blutdruckschwankungen, einer höhten Infektanfälligkeit oder in Stoffwechselentgleisungen äußern.

\section{Yoga in der Schwangerschaft}

\section{Allgemeine Wirkung von Yoga}

Schon seit Jahrzehnten ist den unterschiedlichsten Studien zu entnehmen, dass Yoga körperlich zu einer erhöhten Effizienz führt. Das Herzkreislaufsystem stabilisiert sich, die Lungenfunktion verbessert sich in allen Parametern. Der Stoffwechsel wird stabilisiert, die Serumkonzentration von Cholesterin gesenkt, von Protein erhöht. Die Katecholamine werden vermindert synthetisiert und beschleunigt abgebaut. Der Blutzuckerspiegel gesenkt. Auch das muskuloskelettale System wird durch eine Verbesserung der neuromotorischen Koordination und vertiefter Muskelrelaxation effektiver und elastischer [8]. Die immunologische Wirkung der Yogapraxis lässt sich ebenfalls zunehmend experimentell belegen: Yoga führt zu einer reduzierten Entzündungsneigung und einem stabilisierten Immunstatus [12][17].

Psychisch unterstützt Yoga die Schwangere, indem es die Selbstwahrnehmung verbessert, Ängstlichkeit und Depressivität vermindert und die Stresstoleranz erhöht [8]. Das äußert sich auch in einer schmerzärmeren Geburt [7].
Eine ayurvedische Beratung zu Ernährung und Lebensweise wird oft zur Unterstützung in die Therapie einbezogen, um den Stoffwechsel weiter zu stabilisieren, indem der Hunger respektiert wird und die Gewebe durch eine frische, einfache, feuchte und sanft gewürzte Ernährung genährt werden. Das Prinzip der Reinheit wird gefördert über Rhythmus und Regelmäßigkeit, einem Verzicht auf Genussgifte und übermäßige Stimulation sowie einer Vereinfachung des Lebensstils.

\section{Allgemeine Aspekte der Yogapraxis in der Schwangerschaft}

Yoga ist keine einfache Gymnastik. Man sollte sich gerade in der Schwangerschaft nicht einfach selbst Yoga beibringen. Yogalehrer ist ein Beruf, der vom Bund der Deutschen Yogalehrer (BDY), der Europäischen Yoga Union (EYU) oder anderen Yogaverbänden standardisiert ist und eine mehrjährige Ausbildung umfasst. Ein Yogatherapeut ist ein Yogalehrer, der zusätzlich zu seiner Yogalehrerausbildung eine ausführliche medizinisch-therapeutische Ausbildung absolviert hat. Hier gibt es ebenfalls Standardisierungen der Ausbildungsqualität, z. B. durch die Deutsche Gesellschaft für Yogatherapie (DeGYT) oder durch die International Association of Yoga Therapy (IAYT). Die jeweiligen Websites informieren bei der Suche nach geeigneten Yogalehrern oder -therapeuten.

\section{„Von da an sollte eine schwangere Frau von Beischlaf, körperlichen Übungen, Überessen, Hungern, Schlaf am Tag, Wachen in der Nacht, Trau- ern, Reisen auf Fahrzeugen, Angst und tiefer Hocke Abstand halten und nicht sich einfetten etc., Schröpfen und keine natürlichen Bedürfnisse unterdrücken.“Su. Sa. Sha 3: 35}

asana und pranayama

In den ersten Monaten sollten Schwangere nacheinander alle später für die Geburt benötigten Atemtechniken (pranayama) lernen. Diese sollten während der Schwangerschaft durch konsequentes Üben selbstverständlich werden, sodass die werdende Mutter während der Geburt leicht auf sie zurückgreifen kann.

\section{Erstes Trimenon}

Die asanas, die im ersten Trimenon geübt werden, kräftigen vor allem die Rumpf- und Beckenmuskulatur. Dafür kommen zum Beispiel tadasana, ushtrasana, utkatasana, kati-cakrasana und mulabandha infrage. Dadurch lassen sich Rückenschmerzen, die sich oft im Laufe der Schwangerschaft einstellen, vermeiden. Zudem werden Herz und Kreislauf trainiert und so auf die kommende Mehrbelastung vorbereitet. 


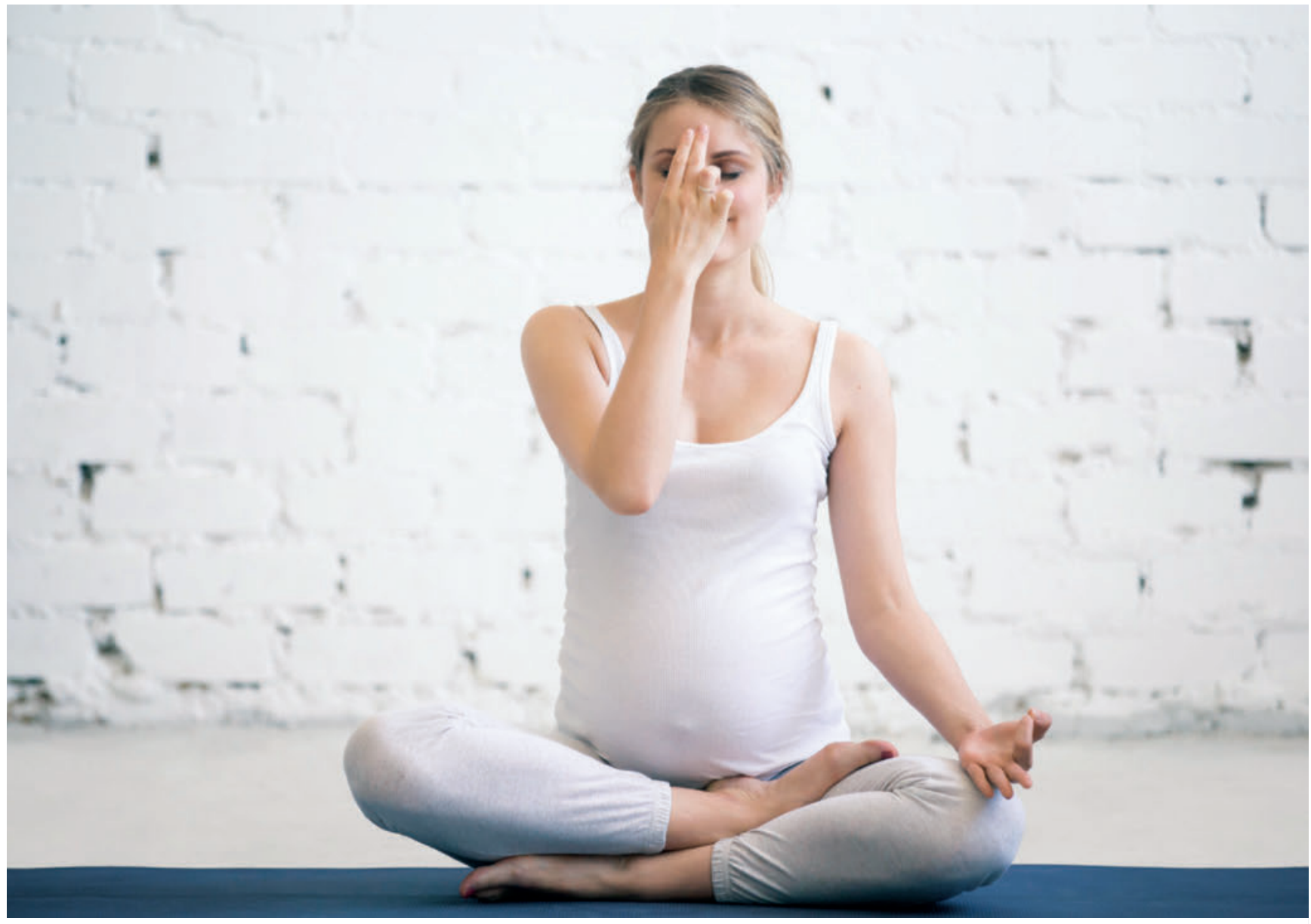

- Abb. 2 Nadi-shodhana-pranayama, die Wechselatmung, kann bei pränatalen Depressionen und Ängstlichkeit eingesetzt werden. @ fizkes/ Adobe Stock; nachgestellte Situation

Aus ayurvedischer Sicht stimulieren diese Übungen die Verdauung und beugen einer Verstopfung vor. Atemübungen, pranayama, gleichen aus. Sie verbessern und vertiefen die Atmung und regen den Stoffwechsel an. Man beginnt meist mit der tiefen Bauchatmung und Atembeobachtung, führt dies weiter zu einem nadishodhana-pranayama ohne kumbhaka. Bei im Yoga bereits fortgeschrittenen Schwangeren kann auch ujjayi- und shitkari-pranayama geübt werden. Dabei geht es nicht um ein schnelles Fitnessprogramm. Besonders wenn eine Schwangere vor ihrer Schwangerschaft keine sportliche Aktivität betrieben hat, sollte sie sanft und vorsichtig mit der Yogapraxis anfangen.

\section{Zweites Trimenon}

Im zweiten Trimenon werden die asanas etwas eingeschränkt. Alle Übungen, die auf den Bauch drücken oder sehr kreislaufbelastend sind, werden nicht mehr in die Praxis einbezogen. Geeignete asanas sind z. B. parvatasana, gomukhasana und marjaryasana. Die Atemübungen werden weiter vertieft, z. B. kann bhramari-pranayama die Atemtiefe fördern und vegetativ entspannen.

\section{Drittes Trimenon}

Gegen Ende der Schwangerschaft steht beim Yoga die Geburtsvorbereitung im Vordergrund mit entspannenden und den Beckenboden dehnenden Übungen. In dieser Phase kann z. B. utthasana eingeführt werden. Dazu bleibt die weitere Perfektionierung der Atemtechniken. Die hechelnden Atemformen und die tiefe Bauchatmung mit kumbhaka dienen der direkten Geburtsvorbereitung.

Es ist besonders wichtig, dass eine Schwangere sich in dieser Phase bei körperlichen Übungen nicht übernimmt. Während die Schwangerschaft weiter fortschreitet, muss sie sich selbst stets beobachten und jeder Überlastung, auch im Alltag, aus dem Weg gehen, um vorzeitige Wehen zu verhindern. Eine im Yoga erfahrene Schwangere kann ihre normale Übungspraxis im ersten Trimenon beibehalten. In dieser Phase sollte sie ihre Übungsfolgen aber graduell auf asanas mit wenig abdomineller Belastung umstellen [8].

\section{Tiefenentspannung}

Für eine stabile und komplikationslose Schwangerschaft und für den reibungslosen Ablauf der Geburt ist es förderlich, wenn Schwangere lernen, sich schnell und tief zu 


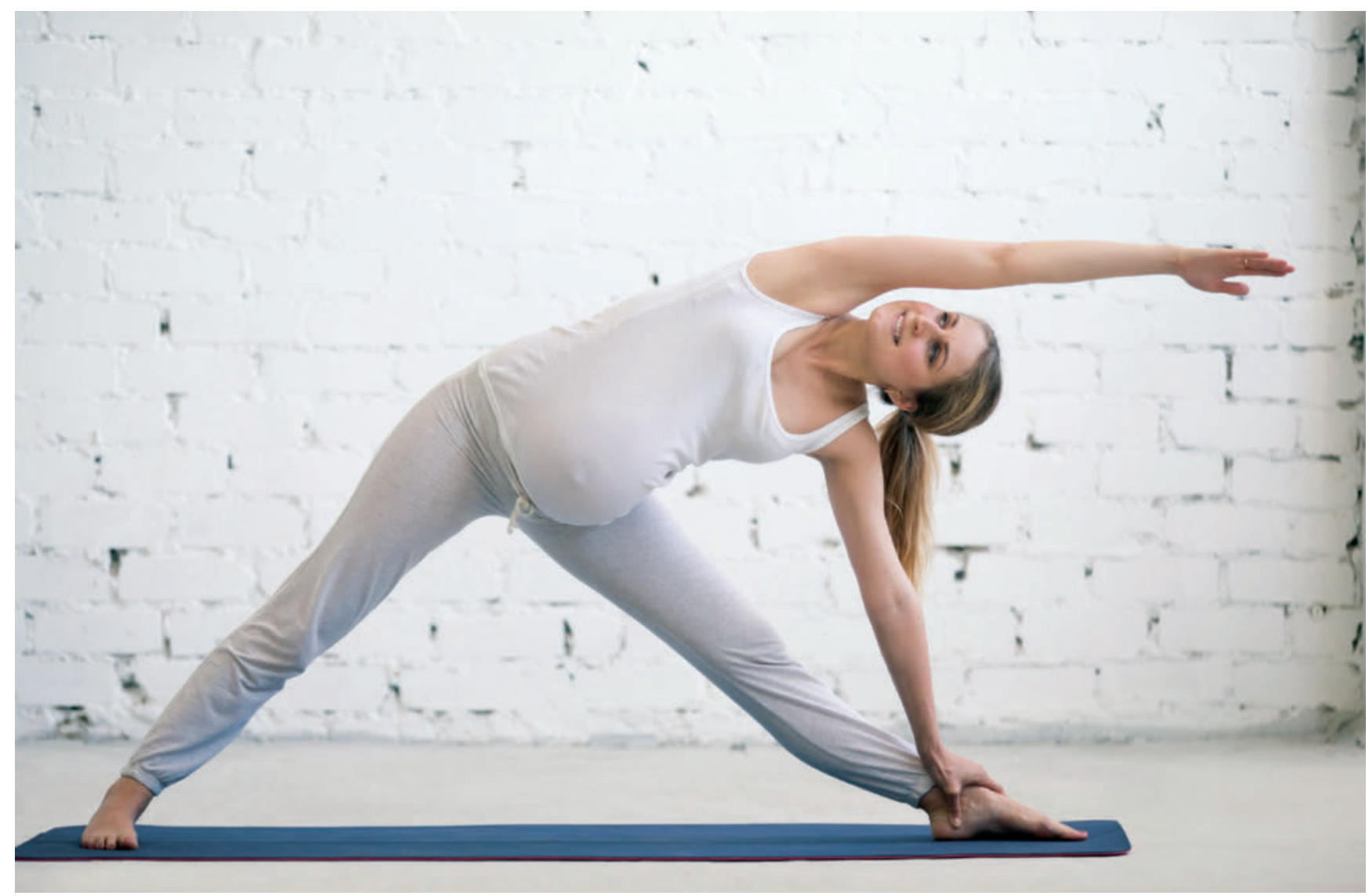

Abb. 3 Utthita Trikonasana, das Dreieck, kann bei Gestationsdiabetes geübt werden. @ fizkes/Adobe Stock; nachgestellte Situation

entspannen. Stressbedingte Störungen wie Schwangerschaftshypertonie oder vorzeitige Wehen können so verhindert werden. Unter der Geburt profitieren Schwangere ebenfalls von Entspannungsverfahren. Die Gebärende kann in den Pausen zwischen den Presswehen besser neue Kraft schöpfen und steht die Geburt leichter durch. Selbst den späteren Aufgaben als Mutter ist sie leichter gewachsen, wenn sie gelernt hat, sich innerhalb kürzester Zeit zu regenerieren.

In verschiedenen Studien zeigt sich, dass Yoga in der Schwangerschaft eine leicht umsetzbare und sichere Therapie ist, selbst bei Hochrisikoschwangerschaften sinnvoll eingesetzt werden kann [1][15][4][3] und die Geburt erleichtert [7].

\section{Therapeutischer Yoga bei Anpassungs- störungen in der Schwangerschaft}

Da die physiologische Wirkung von Yoga zusammengefasst werden kann, als eine Steigerung der Effizienz der funktionellen Systeme und damit einer Verbesserung der Toleranz gegenüber einer Veränderung, ist das Yoga ein geeignetes Instrument in der therapeutischen Begleitung von Anpassungsstörungen während der Schwangerschaft. Dabei wird die Art der Yogapraxis in Abhängigkeit der Art der Anpassungsstörung variiert. Im Folgenden wird beispielhaft das Vorgehen bei Frühgestosen, Rückenschmerzen und bei pränataler Depression erläutert.

\section{Frühgestosen}

Frühgestosen sind Störungen der Adaptation in der Frühgravidität. Sie äußern sich durch morgendliche Übelkeit und Erbrechen, Müdigkeit, ggf. Schwindel und arterielle Hypotonie.

Aus ayurvedischer Sicht ist die Störung des Gleichgewichts zwischen abwärts- und aufwärtsstrebenden vata bei mangelndem ojas (Immunpuffer) die Ursache dieser Symptome.

\section{Yogische Therapie}

Im Yoga setzt man hier eine sehr sanfte Form mit Beobachtung der Atmung und sanften körperlichen Übungen ein. Ideal ist mehrfach am Tag eine kleine Übungspraxis mit Standübungen, Streckübungen, sanftem Drehen und Körperwahrnehmung. Die Atmung sollte dabei vertieft und das Vegetativum über die Wechselatmung ausgeglichen werden. Achtsamkeitsübungen, Mantras und Meditationen können in den Tag eingestreut werden. Studien legen nahe, dass Yoga den Stress von Schwangeren reduziert [11][4]. Damit können Adaptationsprozesse leichter vonstattengehen. 
Dazu werden kleinere, warme Mahlzeiten wie „Magenstreichler" empfohlen, primär süß und leicht gewürzt, um den Stoffwechsel zu stabilisieren.

\section{Rückenschmerzen}

Rückenschmerzen entstehen in der Schwangerschaft durch das zunehmende Körpergewicht bei veränderter Gewichtsverteilung, vermehrtem vorderen Überhang auf der einen Seite und einer hormoninduzierten Bandlaxität auf der anderen Seite. Bewegungsmangel und Fehlhaltung fördern diese Problematik.

Aus ayurvedischer Sicht entstehen Rückenschmerzen nicht nur aufgrund einer biomechanisch veränderten Situation, sondern werden energetisch durch eine vermehrte Reizung des dosha vata im muskuloskelettalen System erklärt.

\section{Yogische Therapie}

Ziel der Therapie ist die Förderung einer stabilen Körperhaltung. Eine Förderung der Beweglichkeit ist in dieser Situation nicht indiziert. Daher wird ein kräftigender Yoga geübt. Der Stand der Füße und Knie, des Beckens und die Aufrichtung der Wirbelsäule wird über Wahrnehmungsübungen und leichte Stellungsveränderungen gefördert, die Kräftigung erfolgt durch Haltungen gegen die Schwerkraft. Die Atmung ist zur Stabilisation der Wirbelsäule ebenfalls essentiell. Tiefe Atmungsformen mit leichtem Halten des Atems fördern die Haltung und die Rumpf- und Beckenbodenmuskulatur.

Die Behandlung von Rückenschmerzen mit Yoga und anderen nichtpharmakologischen Methoden wird inzwischen auch in der Leitlinie des American College of Physicians empfohlen [16]. Rückenschmerzen in der Schwangerschaft werden ebenfalls effektiv nichtpharmakologisch mit Yoga behandelt [10][14].

\section{Pränatale Depression}

Die emotionale Ausgeglichenheit kann während der Schwangerschaft einerseits durch die Veränderungen im Endokrinium, andererseits durch die veränderten Aussichten des Lebens verloren gehen. Manche Frauen reagieren mit Depression oder Ängstlichkeit. Auch hier sind ayurvedisch gesehen nicht nur hormonelle und psychische Faktoren ursächlich, sondern ein energetisches Ungleichgewicht der doshas und der agnis, das einerseits zur emotionalen Instabilität im Sinn von Angstsymptomen, andererseits zur Schwäche von agni mit Bildung von ama und kapha führen kann, was sich als Depression äußern kann.

\section{Yogische Therapie}

Die yogische Therapie zielt auf den Ausgleich und die Förderung der agnis. Ein achtsames Konzept von asanas mit stabilisierenden, fordernden und entspannenden Elementen ist indiziert. Pranayama-Übungen sollten sowohl reinigend als auch ausgleichend sein, wie etwa nadi-shodhana-pranayama, die Wechselatmung. Auch Affirmationen, Mantras und Visualisationen sind bei der Therapie sinnvoll. Yoga wurde in mehreren Studien über Depressionen während der Schwangerschaft untersucht und als sinnvolle und effektive Behandlungsmethode bewertet [2][5][3].

Ernährungsmedizinisch wird dies durch eine agni-stärkende Ernährung unterstützt. Mental stabilisierende und stoffwechselfördernde Kräuter werden dem Essen beigesetzt.

\section{Therapeutischer Yoga bei schweren Erkrankungen während der Schwangerschaft}

Yoga wird nicht nur zur Adaptation eingesetzt, sondern auch in für Mutter und Kind vital gefährlichen Erkrankungen während der Schwangerschaft. Stets wird dabei der Yoga entsprechend der Ziele verändert. Im Folgenden wird 
der Einsatz von Yoga beispielhaft bei Abortus imminens, Spätgestose sowie Schwangerschaftsdiabetes vorgestellt.

\section{Abortus imminens}

Bei einem drohenden Abort kommt es darauf an, den Prozess der Blutung zum Stoppen zu bringen, ohne dass die Frucht Schaden nimmt. Aus ayurvedischer Sicht muss apana-vayu kontrolliert und der Embryo gut genährt werden.

\section{Yogische Therapie}

Neben der Bettruhe werden in der Yogatherapie betont die feinen Techniken geübt. Die tiefe Atmung mit Verlängerung der Ausatmung beruhigt das vegetative Nervensystem. Diese wird mit Visualisationen verbunden, z. B. der Vorstellung eines warmen Lichts im Becken, um das System zu energetisieren und gleichzeitig zu beruhigen. Mantras werden getönt, um den ganzen Körper in eine ruhige Frequenz zu bringen [6].

In der Kashyapasamhita wird besonders das varanabandha gepriesen, ein spezielles Mantra zur Prophylaxe eines Aborts. Mantras bestehen aus Klangabfolgen, die repetitiv getönt werden. Im vedischen Denken geht man davon aus, dass sich die Schwingung des Mantras auf den Körper überträgt und dadurch dessen Funktion beeinflusst. Das varanabandha führt über seine speziellen Frequenzen zu einem Schutz des Ungeborenen und einer Stabilisation der Frucht in utero. Daher darf dieses Mantra nur bis zum achten Monat eingesetzt werden, danach ist es kontraindiziert, um die Geburt nicht zu stören. Eine aktuelle Studie zeigt, dass sich die utero-feto-plazentale Zirkulation durch Yoga verbessern lässt [18], was die Stabilisation der Frucht in utero fördert.

Im Ayurveda wird die Schwangerschaft nicht nur über eine leicht verdauliche warme Ernährung gestützt, sondern auch über eine Vielfalt verschiedener Phytotherapeutika, die einerseits die Frucht nähren, andererseits den vata ausgleichen und außerdem die Blutung stoppen.

\section{Spätgestose}

Eine Spätgestose ist die hypertensive Erkrankung in der Schwangerschaft (HES). Sie kann auch als EPH-Gestose kombiniert mit Ödemen und Proteinurie auftreten. Spätgestosen sind gefährliche Erkrankungen, aus denen sich ein HELLP-Syndrom oder eine Eklampsie entwickeln können mit deutlich gesteigerter perinataler Sterblichkeit.

Im ayurvedischen Verständnis kommt es bei einer Spätgestose zunächst zu einer vata-Störung, die dann verschiedene funktionelle Systeme wie das Lymphsystem, das Blutsystem, das Muskelsystem und das Bindegewebssystem mit den Nieren belastet.

Yogische Therapie

Im Yoga geht es nicht nur darum, sich zu schonen und in Linksseitenlage zu liegen. Yoga wird als sanfte Form der Be- wegung geübt, die die Muskeln achtsam mobilisiert. Zentral sind hier - wie beim Abortus imminens - die feineren yogischen Techniken, die über die Atmung das Vegetativum stabilisieren, über Mantras, Autosuggestion und Visualisation Körper und Geist genügend Stabilität geben, um die vegetative Regulation wieder zu harmonisieren.

Eine neuere Studie vergleicht die Entwicklung der Thrombozyten und der Harnsäure während der Schwangerschaft in einer yogapraktizierenden und einer yogafreien Gruppe. Es zeigt sich, dass die physiologische Anpassung der Thrombozyten und Harnsäurewerte unter Yoga verbessert sind. Es wird diskutiert, dass aus dem gleichem Grund Yoga gerade bei Hochrisikoschwangerschaften und Eklampsie eingesetzt werden sollte [9][19]. Schwangerschaftshochdruck lässt sich mit Yoga leicht und sehr effektiv behandeln, was seit den 1980er-Jahren bekannt ist [13].

Ayurvedisch wird die yogische Therapie mit frischer, warmer, leicht verdaulicher Ernährung gekoppelt, die das Verdauungssystem nicht belastet. Eine Gruppe von ayurvedischen Kräutern stabilisiert die Schwangerschaft, stärkt das Mark und damit das neurovegetative Gleichgewicht.

\section{Gestationsdiabetes}

Diese Stoffwechselstörung tritt in der Spätschwangerschaft vermehrt auf, da dann die Insulinempfindlichkeit sinkt und die Stoffwechselleistung insgesamt steigt.

Aus ayurvedischer Sicht ist diese Störung durch eine Schwäche der agnis, der Verdauungsfeuer, verursacht, die bei der Zunahme an Substanz die vermehrte Ansammlung von kapha und ama nicht verstoffwechseln können.

\section{Yogische Therapie}

Das Ziel der yogischen Therapie ist hier ein anderes. Es soll nicht nur vata regulieren, sondern agni stärken und ama verdauen. Dafür wird ein für die Spätschwangerschaft eher ungewöhnlich aktiver Yoga empfohlen. Dieser besteht aus asanas, die das System fordern, ohne es zu überfordern. Streckungen, Drehungen und Seitneigen sind indiziert. Atemübungen sind nicht nur beruhigend, sondern primär aktivierend mit eher zügigen einatembetonten Atmungsformen. Dabei muss sehr achtsam vorgegangen werden, um eine Überstimulierung zu vermeiden und nicht vorzeitige Wehen auszulösen. Achtsamkeit ist im therapeutischen Yoga noch wichtiger als im prophylaktisch eingesetzten Yoga. In einer aktuellen Studie zeigt sich, dass Yoga den Blutzucker auch bei Gestationsdiabetes senkt [20].

Im Ayurveda werden sanft agni stärkende Kräuter sowie leichte, bittere Kräuter eingesetzt. Klare Brühen und Suppen helfen agni, wieder rein und klar zu brennen. In der Schwangerschaft muss beachtet werden, dass richtiges Fasten kontraindiziert ist. 


\section{Zusammenfassung}

Eine Begleitung der Schwangerschaft und Geburt mit Yoga kann sowohl zur Prophylaxe als auch als Therapie von Adaptationsstörungen und Erkrankungen eingesetzt werden. Alle Aspekte des Yoga können dabei angewandt werden. Im therapeutischen Kontext wird Yoga in Abhängigkeit von den therapeutischen Zielen modifiziert und individualisiert geübt. Studien legen nahe, dass Yogatherapie in der Schwangerschaft nicht nur eine sichere, sondern auch eine effiziente Therapieoption ist. Der therapeutische Yoga entspringt dem Hintergrund des Ayurveda. Daher können auch einfache ayurvedische Empfehlungen in ein Yogatherapiekonzept für Schwangere impliziert werden.

\section{Interessenkonflikt}

Die Autorin gibt an, dass kein Interessenkonflikt besteht.

\section{Autorin}

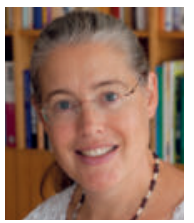

\section{Hedwig H. Gupta}

Fachärztin für Orthopädie und Rheumatologie, Ayurveda, therapeutisches Yoga, Akupunktur, manuelle Medizin. Vorstandsmitglied von DÄGAM und DeGYT, Leiterin der vidya sagar Akademie für Ayurveda und Yogatherapie.

\section{Korrespondenzadresse}

Dr. med. Hedwig H. Gupta

Seestr. 5

71638 Ludwigsburg

E-Mail: info@dr-gupta.de

\section{Literatur}

[1] Babbar S, Shyken J. Yoga in pregnancy. Clin Obstet Gynecol 2016; 59: 600-12

[2] Battle CL, Uebelacker LA, Magee SR et al. Potential for prenatal yoga to serve as an intervention to treat depression during pregnancy. Womens Health Issues 2015; 25: 134-41

[3] Bershadsky S, Trumpfheller L, Kimble HB et al. The effect of prenatal Hatha yoga on affect, cortisol and depressive symptoms. Complement Ther Clin Pract 2014; 20: 106-13

[4] Deshpande CS, Rakhshani A, Nagarathna R et al. Yoga for high-risk pregnancy: a randomized controlled trial. Ann Med Health Sci Res 2016; 3: 341-4

[5] Gong $\mathrm{H}, \mathrm{Ni} \mathrm{C}$, Shen $\mathrm{X}$ et al. Yoga for prenatal depression: a systematic review and meta-analysis. BMC Psychiatry 2015; 15: 14
[6] Hayase M, Shimada M. Effects of maternity yoga on the autonomic nervous system during pregnancy. J Obstet Gynaecol Res 2018; 44: 1887-1895

[7] Jahdi F, Sheikhan F, Haghani $\mathrm{H}$ et al. Yoga during pregnancy: The effects on labor pain and delivery outcomes (a randomized controlled trial). Complement Ther Clin Pract 2017; $27: 1-4$

[8] Jain MD, Hepp HH. Yoga als adjuvandte Therapie. Stuttgart: Hippokrates; 1998

[9] Jayashree R, Malini A, Rakhshani A. Effect of the integrated approach of yoga therapy on platelet count and uric acid in pregnancy: a multicenter stratified randomized single-blind study. Int J Yoga 2013; 6: 39-46

[10] Kinser PA, Pauli ], Jallo $N$ et al. Physical activity and yogabased approaches for pregnancy-related low back and pelvic pain. J Obstet Gynecol Neonatal Nurs 2017; 46(3): 334-346

[11] Kusaka M, Matsuzaki M, Shiraishi M et al. Immediate stress reduction effects of yoga during pregnancy: one group prepost test. Women Birth 2016; 29(5): e82-e88

[12] Lim SA, Cheong KJ. Regular yoga practice improves antioxidant atatus, immune function, and stress hormone releases in young healthy people: a randomized, double-blind, controlled pilot study. J Altern Complement Med 2015; 21: $530-8$

[13] Little BC, Hayworth J, Benson P et al. Treatment of hypertension in pregnany by relaxation and biofeedback. Lancet 1984; i: 865-8

[14] Martins RF, Pinto e Silva JL. Treatment of pregnancy-related lumbar and pelvic girdle pain by the yoga method: a randomized controlled study. J Altern Complement Med 2014; 20: 24-31

[15] Polis RL, Gussman D, Kuo YH. Yoga in pregnancy: an examination of maternal and fetal responses to 26 Yoga postures. Obstet Gynecol 2015; 126: 1237-41

[16] Qaseem A, Wilt T], McLean RM et al. Clinical guidelines committee of the american college of physicians. Noninvasive treatments for acute, subacute, and chronic low back pain: a clinical practice guideline from the american college of physicians. Ann Intern Med 2017; 166: 514-530

[17] Rajbhoj PH, Shete SU, Verma A et al. Effect of yoga module on pro-inflammatory and anti-inflammatory cytokines in industrial workers of lonavla: a randomized controlled trial. J Clin Diagn Res 2015; 9: CC01-5

[18] Rakhshani A, Nagarathna R, Mhaskar R et al. Effects of yoga on utero-fetal-placental circulation in high-risk pregnancy: a randomized controlled trial. Adv Prev Med 2015: 373041

[19] Rakhshani A, Nagarathna R, Mhaskar R et al. The effects of yoga in prevention of pregnancy complications in high-risk pregnancies: a randomized controlled trial. Prev Med 2012; 55: 333-340

[20] Youngwanichsetha S, Phumdoung S, Ingkathawornwong T. The effects of mindfulness eating and yoga exercise on blood sugar levels of pregnant women with gestational diabetes mellitus. Appl Nurs Res 2014; 27: 227-30

Bibliografie

DOI https://doi.org/10.1055/a-0785-5987

EHK 2018; 67: 345-351

(c) MVS Medizinverlage Stuttgart GmbH \& Co. KG

ISSN 0014-0082 